Rosilene da Conceição R. Moreira 1 José Manuel Macário Rebêlo 1 Mônica Elinor Alves Gama 1 Jackson Maurício L. Costa 1

\section{Nível de conhecimentos sobre Leishmaniose Tegumentar Americana (LTA) e uso de terapias alternativas por populações de uma área endêmica da Amazônia do Maranhão, Brasil}

\author{
Awareness of American tegumentary leishmaniasis \\ (ATL) and use of alternative therapies in an \\ endemic area in the Amazon Region in the State \\ of Maranhão, Brazil
}

1 Núcleo de Patologia Tropical e Medicina Social, Departamento de Patologia, Universidade Federal do Maranhão. Praça Madre Deus 2, São Luís, MA 65025-560, Brasil.

\begin{abstract}
The level of knowledge concerning American tegumentary leishmaniasis (ATL) and use of alternative therapies was evaluated in five rural communities in Buriticupu, Maranhão State, Brazil. The study lasted from September 1997 to January 1998. Local inhabitants answered an interview on housing conditions, epidemiological aspects, prevention, standard clinical treatment, and alternative therapies. The study population was 378 (19\%) out of a total of 1,980 inhabitants, from Sexta Vicinal (35), Quinta Vicinal (63), Trilha 410 (96), Vila União (85), and Buritizinho (99). Of the interviewees, $72 \%$ had little knowledge of ATL transmission, 96\% had heard something about the disease from friends, and 60.7\% knew ATL by the local term "lésh". Glucantime was the most familiar drug for treatment, while 29.6\% referred to use of herbal remedies on the ulcers. Citrus limon (lemon) was the plant most frequently used, and $15.4 \%$ of the interviewees used it as a powder spread on the wound. We conclude that the population had little correct knowledge of ATL in the five areas studied, especially with regard to prevention and treatment.
\end{abstract}

Key words Leishmaniasis; Amazonian Ecosystem; Alternative Therapies; Knowledge

Resumo Realizou-se estudo visando identificar e comparar que conhecimentos básicos e uso de terapias alternativas relativos à Leishmaniose Tegumentar Americana (LTA), têm populações residentes em cinco áreas rurais (Sexta Vicinal, Quinta Vicinal, Trilha 410, Vila União e Buritizinho) pertencentes ao município de Buriticupu, Maranhão. No período de setembro de 1997 a janeiro de 1998, aplicou-se questionários com perguntas abertas e fechadas, abordando aspectos epidemiológicos, modos de prevenção, clínica e terapêutica alternativa. A população estudada foi de 378 (19\%) indivíduos de um total de 1980 habitantes. Na Sexta Vicinal (35 indivíduos), Quinta Vicinal (63), Trilha 410 (96), Vila União (85) e Buritizinho (99). Dos entrevistados, 72\% tinham poucos conhecimentos dos modos de transmissão, 96,9\% já ouviram falar da doença, a maioria obteve informações com amigos, 60,7\% conheciam a LTA como lésh. O Glucantime foi a droga mais citada para o tratamento, 29,6\% relataram uso de plantas no local da lesão. O Citrus limon (limão) foi a planta mais citada $(15,4 \%)$ dos entrevistados, o modo de uso mais freqüente era o pó espalhado sobre a lesão. Conclui-se que o nível de conhecimento sobre LTA foi incipiente, principalmente na prevenção e terapêutica, situação similar nas cinco áreas estudadas.

Palavras-chave Leishmaniose; Ecossistema Amazônico; Terapias Alternativas; Conhecimento 


\section{Introdução}

Apesar da Leishmaniose Tegumentar Americana (LTA) encontrar-se amplamente distribuída nas Américas, entender seus aspectos epidemiológicos, constitui-se em tarefa extremamente complexa; requer a obtenção de um conjunto de informações que possam ser úteis para uma eventual proposta de medidas de controle, levando-se em consideração as peculiaridades de cada região (Grimaldi Jr. et al., 1989; WHO, 1990).

Netto et al., (1985), observaram que grande parte da população da Região de Três Braços, Bahia, área endêmica de LTA, detinha um entendimento razoável sobre alguns aspectos epidemiológicos e do tratamento da doença, sendo freqüente o uso de vários preparados alternativos feitos pela população à base de ervas como medida terapêutica. Porém, demonstravam desconhecimento nos aspectos relacionados à etiologia e prevenção da doença.

Apesar dessa prática parecer comum entre as populações das áreas endêmicas, o seu real valor científico ainda não foi estabelecido. Mesmo em se tratando de lesões produzidas por Leishmania braziliensis, parasito responsável por quadros graves de lesões mucosas, vários preparados à base de plantas foram usados pela população de Três Braços, Bahia, entre elas o Anacardium occidentale (cajueiro branco) como uma das condutas no tratamento da LTA na região (França et al., 1996; Netto et al., 1985).

França et al., (1996), em estudo experimental, observaram que o extrato do A. occidentale proveniente da região de Três Braços apresentou atividade in vitro quando testado contra as formas promastigotas de Le. braziliensis, isoladas de pacientes portadores de LTA provenientes da mesma área endêmica. Quando usou o referido extrato por via tópica em lesões no hamster (Mesocricetus auratus) não obteve bons resultados.

Oficialmente, as drogas consideradas de escolha no tratamento da LTA são os antimoniais pentavalentes (Antimoniato-N-metil-glucamina e o Estibogluconato de sódio), embora saibamos que nas lesões cutâneas da LTA é freqüente ocorrer cicatrização espontânea, como demonstraram Marsden (1986) e Costa et al., (1990).

No Estado do Maranhão, temos poucos estudos sobre os aspectos ecoepidemiológicos da LTA. Sua localização geográfica, biodiversidade, torna-o importante para estudos concernentes a este aspecto da doença. Apesar de sua importância no Estado, desconhece-se os métodos alternativos utilizados pela população no tratamento da LTA. Sabe-se, todavia, que na Amazônia do Maranhão, o Município de Buriticupu é o de maior prevalência da doença e que os conhecimentos dessa população sobre alguns aspectos da LTA são incipientes, principalmente em relação aos preparados feitos à base de plantas como alternativas usadas no tratamento da lesão (Costa et al., 1998; Moreira et al., 1998; Rebêlo et al., 2000).

O presente estudo, visa identificar os conhecimentos que determinadas populações da região de Buriticupu apresentam sobre a LTA e as alternativas terapêuticas usadas na referida doença.

\section{Material e métodos}

Realizou-se um estudo prospectivo, no período de setembro de 1997 a janeiro de 1998, em cinco áreas endêmicas para LTA, no Município de Buriticupu, Maranhão. Os povoados selecionados para o estudo foram: Sexta Vicinal, Quinta Vicinal e Trilha 410, localidades que fizeram parte desde o início da criação do Município de Buriticupu (1972) e, também, localidades criadas mais recentemente (década de 90: Buritizinho e União). Todas acompanhadas por equipes de pesquisadores da Universidade Federal do Maranhão (UFMA), no que diz respeito à LTA, desde suas criações (Costa et al., 1998; Rebêlo et al., 2000; Silva et al., 1979, 1981).

O Município de Buriticupu, localizado na Amazônia do Maranhão, entre 4o27'22" e 4o30' 00” LS e 46³5'27” e 4654'03” LW, apresenta população atual de 30.000 habitantes (IBGE, 2000), distando $450 \mathrm{~km}$ de São Luís. Tem como principais atividades a agricultura e a pecuária. (Costa et al., 1998; Rebêlo et al., 2000; Silva et al., 1979). Quando de sua criação, visava fixar dez mil famílias de agricultores, tendo sido agrupadas em organizações urbanas com três categorias: povoado, núcleo administrativo avançado e agrovilas. A situação atual tem como ponto principal de estrangulamento, a quebra da filosofia original de ocupação ordenada da região (Costa et al.,1998; Silva et al., 1979).

O trabalho de campo junto às comunidades foi realizado por uma equipe de profissionais da área de saúde, pertencente à UFMA, devidamente treinados quanto à utilização do material e com relativo conhecimento das áreas. Por meio de entrevista individual com o responsável pela residência no momento da visita, procedeu-se aplicação de uma ficha-questionário composta de perguntas abertas e fechadas, das quais constavam dados relativos à identificação, condições de moradia, características epi- 
demiológicas e conhecimentos sobre aspectos epidemiológicos, preventivos, clínicos, terapêuticas convencionais e alternativas usadas na LTA.

A população de estudo foi composta por 378 indivíduos (correspondendo a 378 famílias, cuja população era de 1.980 habitantes), sendo entrevistados 85 indivíduos no povoado de União, 35 em Sexta Vicinal, 63 em Quinta Vicinal, 96 na Trilha 410 e em Buritizinho 99.

Procedeu-se análise estatística dos dados, utilizando-se o teste do Qui-Quadrado para estabelecer diferenças significativas $(p \leq 0,05)$, do programa Epi Info, versão 6.0 (Dean, 1994).

\section{Resultados}

Nas cinco localidades estudadas, foram entrevistados 378 indivíduos, assim distribuídos: Vila União (85), Sexta Vicinal (35), Quinta Vicinal (63), Buritizinho (99) e Trilha 410 (96). Houve predomínio do sexo feminino, faixa etária entre 21 a 40 anos de idade, em todas as localidades. Quanto ao grau de instrução, destacaramse os analfabetos com a seguinte distribuição: Sexta Vicinal (60\%), Vila União $(49,4 \%)$, Trilha 410 (40,6\%), Buritizinho (39,4\%) e Quinta Vicinal $(57,1 \%)$. Em relação à ocupação, houve destaque a função doméstica, seguida de lavrador em todas as localidades estudadas.

Quanto às questões sanitárias, dos 378 entrevistados, 96,6\% não dispunham de banheiro nas casas, $50,8 \%$ responderam que jogavam os dejetos a céu aberto e $30,4 \%$ tinham fossa negra (sentina), 18,3\% apresentavam fossa séptica e 5\% não souberam informar. A água consumida na residência procedia da rede de abastecimento público $(7,9 \%)$, poço coletivo $(63 \%)$, poço próprio $(23,0 \%)$, outra procedência da água ou então não sabiam informar (6\%). Na Tabela 1, pode-se observar a estrutura física das residências dos entrevistados. Nas localidades: Trilha 410 e Buritizinho as casas apresentavam melhor estrutura física, em relação à Sexta Vicinal, Quinta Vicinal e Vila União ( $\mathrm{p}$ 0.0000) predominando cobertura de telha, parede de madeira e piso de cimento.

A Tabela 2, sintetiza as características epidemiológicas das populações: 339(89,7\%) pessoas citaram a presença de animais domésticos no domicílio e peridomicílio. Na Vila União $(89,4 \%)$, Sexta Vicinal $(57,1 \%)$, Quinta Vicinal (79,4\%), Buritizinho (99\%) e Trilha 410 (99\%). Raras foram as citações sobre a presença de animais silvestres, tais como: gambá (Didelphis sp.) e raposa (Cerdocyon sp./Dusicyon sp.). Um total de 190 (50,3\%) pessoas, citaram a presen- ça de insetos, porém somente $99(26,1 \%)$ mostraram prováveis criadouros no domicílio ou próximo de suas casas.

A maioria das residências dos entrevistados situava-se próximo da mata e/ou chiqueiros, galinheiros, estábulos: Vila União $(87,2 \%)$, Sexta Vicinal $(91,5 \%)$, Quinta Vicinal $(95,3 \%)$, Trilha 410 (99,1\%) e Buritizinho (100\%).

Quando avaliou-se a presença de casos de LTA na família, observou-se que 154 (7,7\%) pessoas tiveram história pregressa da doença. Em União 54,1\%, Sexta Vicinal 71,4\%, Quinta Vicinal 47,6\%, Buritizinho 27,3\% e Trilha 410 $(27,1 \%)$. Cerca de $156(41,2 \%)$ entrevistados referiram casos de indivíduos com LTA nas proximidades de suas casas.

A Tabela 3, mostra o nível de conhecimentos dos entrevistados sobre LTA: em Buritizinho $96,9 \%$ já ouviram falar da doença, Vila União (90,6\%), Sexta Vicinal (80\%), Quinta Vicinal $(79,4 \%)$, Trilha $410(89,9 \%)$. Essas informações foram adquiridas pela maioria dos entrevistados através de contato com amigos $(71,7 \%)$, poucas $(5 \%)$ foram recebidas pelos órgãos de comunicação (televisão, rádio). Quanto às escolas e agentes de saúde, somente (3\%) e $(1,1 \%)$ respectivamente, receberam deles algum tipo de informação sobre a doença.

Para a pergunta: Como era conhecido a LTA na região? Teve-se como principal resposta lésh: Vila União (45,9\%), Sexta Vicinal $(37,1 \%)$, Quinta Vicinal (50,8\%), Trilha $410(69,7 \%)$ e Buritizinho $(79,2 \%)$. Menos freqüentes foram boba, ferida braba e ferida brava. A maioria não sabia como a doença era transmitida. Na Trilha $410,27,3 \%$ disseram saber quem transmite a doença, e em Buritizinho (15,6\%). Nessas duas localidades (Trilha 410 e Buritizinho, 55,6\% e $66,7 \%$ respectivamente), acreditavam ser um inseto o vetor, sendo o mais citado "borrachudo" (Simulium sp.).

É durante os meses de chuva (dezembro, janeiro, fevereiro, março) que aparecem a maioria dos casos de LTA na região: Vila União $(57,1 \%)$ dos entrevistados, Sexta Vicinal $(57,1 \%)$, Quinta Vicinal (22,2\%), Trilha 410 (29,3\%) e Buritizinho (26,\%). A grande maioria das pessoas não faz uso de medidas de prevenção contra a doença; 78\% em União, 74,3\% em Sexta Vicinal, 88,9\% em Quinta Vicinal, 86,9\% em Buritizinho e $82,3 \%$ na Trilha 410 . Todavia, os moradores referiram conhecer o Glucantime como a principal medicação utilizada para o tratamento da LTA: Vila União $(27,1 \%)$, Sexta Vicinal $(54,3 \%)$, Quinta Vicinal (50,8\%), Trilha 410 (19,2\%) e Buritizinho (33,3\%). Em seguida vieram os remédios caseiros, Benzetacil, pingo de vela, produtos químicos, instrumentos quentes, pólvora, 
Estrutura física das residências das populações estudadas.

\begin{tabular}{|c|c|c|c|c|c|c|c|c|c|c|}
\hline \multirow{2}{*}{$\begin{array}{l}\text { Estrutura física } \\
\text { da residência }\end{array}$} & \multicolumn{2}{|c|}{ Local 1} & \multicolumn{2}{|c|}{ Local 2} & \multicolumn{2}{|c|}{ Local 3} & \multicolumn{2}{|c|}{ Local 4} & \multicolumn{2}{|c|}{ Local 5} \\
\hline & $f$ & $\%$ & $f$ & $\%$ & $f$ & $\%$ & $f$ & $\%$ & $f$ & $\%$ \\
\hline \multicolumn{11}{|c|}{ Tipo de cobertura } \\
\hline Telha & 9 & 10,6 & 5 & 14,3 & 15 & 23,8 & 76 & 76,8 & 94 & 97,9 \\
\hline Palha & 22 & 25,9 & 4 & 1,4 & 1 & 1,6 & 12 & 12,1 & - & - \\
\hline Madeira & 54 & 63,5 & 26 & 74,3 & 43 & 68,3 & 9 & 9,1 & - & - \\
\hline Laje & - & - & - & - & - & - & - & - & 2 & 2,1 \\
\hline Outros & - & - & - & - & - & - & - & - & - & - \\
\hline Ignorado & - & - & - & - & 4 & 6,3 & 2 & 2,0 & - & - \\
\hline \multicolumn{11}{|l|}{ Tipo de parede } \\
\hline Taipa & 83 & 97,6 & 31 & 88,6 & 51 & 81,0 & 79 & 79,8 & 1 & 1,0 \\
\hline Madeira & - & - & 1 & 2,9 & 5 & 7,9 & 14 & 14,1 & 93 & 96,9 \\
\hline Adobe & 2 & 2,4 & - & - & - & - & - & - & - & - \\
\hline Alvenaria & - & - & - & - & - & - & 1 & 1,0 & - & - \\
\hline Ignorado & - & - & 3 & 8,6 & 7 & 11,1 & 5 & 5,1 & 2 & 2,1 \\
\hline \multicolumn{11}{|l|}{ Tipo de piso } \\
\hline Cerâmica & - & - & - & - & - & - & 1 & 1,0 & - & - \\
\hline Cimento & 10 & 11,8 & 04 & 11,4 & 12 & 19,0 & 32 & 32,3 & 92 & 95,8 \\
\hline Chão batido & 73 & 85,9 & 31 & 88,6 & 49 & 77,8 & 63 & 63,6 & 4 & 4,2 \\
\hline Madeira & - & - & - & - & - & - & 1 & 1,0 & - & - \\
\hline Ignorado & 2 & 2,4 & - & - & 2 & 3,2 & 2 & 2,0 & - & - \\
\hline
\end{tabular}

Chave para identificação das localidades:

Local 1 - União; Local 2 - Sexta Vicinal; Local 3 - Quinta Vicinal; Local 4 - Buritizinho; Local 5 - Trilha 410

Tabela 2

Características epidemiológicas das populações estudadas.

\begin{tabular}{|c|c|c|c|c|c|c|c|c|c|c|}
\hline \multirow{3}{*}{$\begin{array}{l}\text { Características } \\
\text { epidemiológicas }\end{array}$} & \multicolumn{2}{|c|}{ Local 1} & \multicolumn{2}{|c|}{ Local 2} & \multicolumn{2}{|c|}{ Local 3} & \multicolumn{2}{|c|}{ Local 4} & \multicolumn{2}{|c|}{ Local 5} \\
\hline & $f$ & $\%$ & $f$ & $\%$ & $f$ & $\%$ & $f$ & $\%$ & f & $\%$ \\
\hline & 76 & 89,4 & 20 & 57,1 & 50 & 79,4 & 98 & 99,0 & 95 & 99,0 \\
\hline $\begin{array}{l}\text { Animais silvestres } \\
\text { no domicílio ou } \\
\text { próximo dele }\end{array}$ & - & - & - & - & 2 & 3,2 & 1 & 1,0 & - & - \\
\hline $\begin{array}{l}\text { Presença de outros } \\
\text { animais }\end{array}$ & 8 & 9,4 & - & - & 1 & 1,6 & - & - & - & - \\
\hline Mosquitos no domicílio & 47 & 55,3 & 15 & 42,9 & 44 & 69,8 & 44 & 44,4 & 40 & 41,7 \\
\hline Criadouros de mosquitos & 7 & 8,2 & 4 & 11,4 & 58 & 92,1 & 2 & 2,0 & 28 & 29,2 \\
\hline História de LTA na família & 46 & 54,1 & 25 & 71,4 & 30 & 47,6 & 27 & 27,3 & 26 & 27,1 \\
\hline $\begin{array}{l}\text { História de LTA nas } \\
\text { proximidades da casa }\end{array}$ & 34 & 40,0 & 18 & 51,4 & 34 & 54,0 & 35 & 35,4 & 35 & 36,5 \\
\hline $\begin{array}{l}\text { Residência localizada } \\
\text { próxima da mata/ } \\
\text { capoeira e criações } \\
\text { de animais }\end{array}$ & 77 & 87,2 & 32 & 91,5 & 60 & 95,3 & 98 & 99,1 & 96 & 100,0 \\
\hline
\end{tabular}

Chave para identificação das localidades:

Local 1 - União; Local 2 - Sexta Vicinal; Local 3 - Quinta Vicinal; Local 4 - Buritizinho; Local 5 - Trilha 410 
Principais respostas às questões abordadas sobre os aspectos gerais da Leishmaniose Tegumentar Americana (LTA).

\begin{tabular}{|c|c|c|c|c|c|c|c|c|c|c|}
\hline \multirow{2}{*}{$\begin{array}{l}\text { Questões } \\
\text { abordadas }\end{array}$} & \multicolumn{2}{|c|}{ Local 1} & \multicolumn{2}{|c|}{ Local 2} & \multicolumn{2}{|c|}{ Local 3} & \multicolumn{2}{|c|}{ Local 4} & \multicolumn{2}{|c|}{ Local 5} \\
\hline & & $\%$ & $f$ & $\%$ & $f$ & $\%$ & & $\%$ & & $\%$ \\
\hline \multicolumn{11}{|c|}{ Já ouviram falar em LTA? } \\
\hline Sim & 77 & 90,6 & 28 & 80,0 & 50 & 79,4 & 89 & 89,9 & 93 & 96,9 \\
\hline Não & 8 & 9,4 & 7 & 20,0 & 12 & 19,0 & 9 & 9,1 & 2 & 2,1 \\
\hline Não sabe informar & - & - & - & - & 1 & 1,6 & 1 & 1,0 & 1 & 1,0 \\
\hline \multicolumn{11}{|l|}{ Outros nomes para LTA } \\
\hline Lésh & 39 & 45,9 & 13 & 37,1 & 32 & 50,8 & 69 & 69,7 & 76 & 79,2 \\
\hline Ferida brava & 8 & 9,4 & 4 & 11,4 & 7 & 11,1 & 4 & 4,0 & 7 & 7,3 \\
\hline Ferida braba & 9 & 10,6 & 4 & 11,4 & 4 & 6,3 & 3 & 3,0 & 4 & 4,2 \\
\hline Não sabe informar & 25 & 29,4 & 14 & 40,0 & 20 & 31,7 & 21 & 21,2 & 9 & 9,4 \\
\hline \multicolumn{11}{|l|}{$\begin{array}{l}\text { Sabe quem transmite } \\
\text { a LTA? }\end{array}$} \\
\hline Sim & 21 & 24,7 & 12 & 34,3 & 18 & 28,6 & 27 & 27,3 & 15 & 15,6 \\
\hline Não & 64 & 75,3 & 21 & 60,0 & 44 & 69,8 & 71 & 71,7 & 72 & 75,0 \\
\hline Não sabe informar & - & - & - & - & - & - & 1 & 1,0 & 9 & 9,4 \\
\hline \multicolumn{11}{|c|}{ Como é transmitida a LTA? } \\
\hline Mosquito & 19 & 22,4 & 12 & 34,3 & 18 & 28,6 & 20 & 55,6 & 14 & 66,7 \\
\hline Não sabe informar & 65 & 76,5 & 21 & 60,0 & 45 & 71,4 & 16 & 44,4 & 7 & 33,3 \\
\hline \multicolumn{11}{|l|}{$\begin{array}{l}\text { Sabe quais os animais } \\
\text { que apresentam LTA? }\end{array}$} \\
\hline Sim & 17 & 20,0 & 3 & 8,6 & 5 & 7,9 & 12 & 12,1 & 17 & 17,7 \\
\hline Não & 67 & 78,8 & 27 & 77,1 & 56 & 88,9 & 63 & 63,6 & 59 & 61,5 \\
\hline Não sabe informar & 1 & 1,2 & 5 & 14,3 & 2 & 3,2 & 24 & 24,2 & 20 & 20,8 \\
\hline \multicolumn{11}{|c|}{$\begin{array}{l}\text { Fazem uso de medidas } \\
\text { profiláticas contra a LTA? }\end{array}$} \\
\hline Sim & 18 & 21,2 & 9 & 25,7 & 7 & 11,1 & 5 & 5,1 & 12 & 12,5 \\
\hline Não & 67 & 78,8 & 26 & 74,3 & 56 & 88,9 & 86 & 86,9 & 79 & 82,3 \\
\hline \multicolumn{11}{|c|}{ Onde ouviu falar de LTA? } \\
\hline Tv & 3 & 3,5 & - & - & 2 & 3,2 & 3 & 3,0 & 2 & 2,1 \\
\hline Escola & - & - & - & - & - & - & 1 & 1,0 & - & - \\
\hline Amigos & 51 & 60,0 & 25 & 71,4 & 46 & 73,0 & 82 & 82,8 & 67 & 69,8 \\
\hline Agente de saúde & 6 & 7,1 & 3 & 8,6 & 1 & 1,6 & & & & \\
\hline Outros & 15 & 17,6 & 2 & 5,7 & - & - & - & - & 15 & 15,6 \\
\hline Não sabe informar & 10 & 11,8 & 5 & 14,3 & 12 & 19,0 & 12 & 12,1 & 12 & 12,5 \\
\hline \multicolumn{11}{|c|}{ Quanto tempo dura a LTA? } \\
\hline 1 mês & 2 & 2,4 & - & - & 3 & 4,8 & 2 & 2,0 & - & - \\
\hline 2 meses & 1 & 1,2 & - & - & 1 & 1,6 & 2 & 2,0 & 1 & 1,0 \\
\hline Alguns meses & 9 & 10,6 & 3 & 8,6 & 8 & 12,7 & 1 & 1,0 & - & - \\
\hline 1 ano & 6 & 7,1 & 4 & 11,4 & 5 & 7,9 & 1 & 1,0 & - & - \\
\hline Outros & 14 & 16,5 & 11 & 31,4 & 16 & 25,4 & 12 & 12,1 & 3 & 3,1 \\
\hline Não sabe informar & 45 & 52,9 & 17 & 48,6 & 30 & 47,6 & 80 & 80,8 & 92 & 95,8 \\
\hline \multicolumn{11}{|c|}{$\begin{array}{l}\text { Sabe qual a medicação } \\
\text { para tratar a LTA? }\end{array}$} \\
\hline Sim & 23 & 27,1 & 19 & 54,3 & 32 & 50,8 & 19 & 19,2 & 32 & 33,3 \\
\hline Não & 59 & 69,4 & 15 & 42,9 & 31 & 49,2 & 80 & 80,8 & 63 & 65,6 \\
\hline Não sabe informar & 3 & 3,5 & 1 & 2,9 & - & - & - & - & - & - \\
\hline \multicolumn{11}{|c|}{ Conhece o glucantime? } \\
\hline Sim & 36 & 42,4 & 22 & 62,9 & 37 & 58,7 & 39 & 39,4 & 40 & 41,7 \\
\hline Não & 47 & 55,3 & 13 & 37,1 & 26 & 41,3 & 60 & 60,6 & 56 & 58,3 \\
\hline Não sabe informar & 2 & 2,4 & - & - & - & - & - & - & - & - \\
\hline
\end{tabular}

Chave para identificação das localidades:

Local 1 - União; Local 2 - Sexta Vicinal; Local 3 - Quinta Vicinal; Local 4 - Buritizinho; Local 5 - Trilha 410 
sapo torrado, também foram citados como opção terapêutica tópica pelos entrevistados.

Dos 378 entrevistados, $29,6 \%$, relataram o uso de plantas no local da ferida. A Tabela 4, mostra as principais espécies citadas e o seu modo de uso. Vários usavam combinação de duas ou mais plantas, porém, apesar de algumas pessoas relatarem melhora com o uso das plantas no local da ferida, o Glucantime foi o medicamento mais citado como terapêutica da LTA entre os entrevistados.

\section{Discussão}

Considerando-se ser a LTA uma das doenças de maior ocorrência em áreas rurais do Maranhão, realizou-se o presente estudo em localidades do município de Buriticupu que possuem características de endemicidade para a doença (Costa et al., 1998).

Sabendo-se que as localidades Trilha 410 e Buritizinho instaladas há mais de 27 anos, a primeira por receber incentivo do Governo Federal (antiga reserva destinada aos indígenas), a segunda por localizar-se às margens da BR316 (rodovia que liga o Município de Santa Luzia a Açailândia, confluência com a Belém/Brasília), justifica-se sua melhor estrutura física; no entanto, apesar dos benefícios, observou-se precariedade no saneamento básico. Todas as localidades mostraram características de moradia compatíveis com citações da literatura, que afirmam ser a LTA mais incidente em áreas de baixo nível sócio-econômico, com casas de taipa, cobertas de palha e piso de terra batida (Costa, 1986; Costa et al., 1988; Follador et al., 1999).

O predomínio do sexo feminino no estudo é justificado pelo fato dos homens passarem a maior parte do dia na lavoura, retornando, em geral, ao anoitecer. Costume relativamente comum nas áreas endêmicas de LTA e LVA do Brasil. Nota-se ainda, que a mulher desempenha uma dupla jornada de trabalho (dona de casa e lavoura), considerando ser da roça que as populações rurais mantêm sua subsistência, assim como da caça e da pesca, funções onde a participação masculina é mais efetiva. Estes fatos explicam a maior prevalência da LTA em indivíduos que se dedicam a tarefas agrícolas (Costa, 1986; Pessoa \& Barreto, 1948; Silva et al., 1979).

A observação de animais (porcos, cães, galinhas) mesmo sadios, segundo os entrevistados, próximos ao domicílio, representam potenciais reservatórios ou fonte de alimentação para o inseto vetor, como demonstraram Ampuero (1996) e Rebêlo et al., (2000), em áreas endêmicas de LTA. A localização das casas perto da capoeira, permite o aparecimento de insetos que invadem o domicílio e/ou peridomicílio. Apesar de ter sido o borrachudo (Simulium sp.) o mais citado como o provável transmissor para os entrevistados, o "tatuquira" (flebótomo) mosquito que acompanha o tatu (nome popular dado originalmente pelos indígenas da região, que posteriormente a população de um modo geral adotou), encontra-se presente tanto no intra como no peridomicílio, fato preocupante, visto que as pessoas podem adquirir a infecção na sua própria habitação, como demonstraram Ampuero (1996) na região de Corte de Pedra/Bahia e Rebêlo et al., (2000), em Buriticupu/Maranhão.

O período chuvoso foi a época citada como a de maior incidência da doença, coincide com o tempo de plantio e colheita de culturas agrícolas, onde a exposição ao vetor é mais constante pelas populações da região estudada. Tais fatos são similares aos relatados por Costa (1986), na região de mata Atlântica da Bahia,

Tabela 4

Espécies de plantas mais citadas nas localidades estudadas de Buriticupu.

\begin{tabular}{lcll}
\hline Espécies de plantas & $\begin{array}{l}\text { f de indivíduos que } \\
\text { citaram as plantas }\end{array}$ & Nome comum & Modo de uso das plantas \\
\hline Citrus limon & 28 & Limão & $\begin{array}{l}\text { Pó da folha (espalhado pela ferida } \\
\text { e infusão das folhas sobre a ferida) }\end{array}$ \\
$\begin{array}{l}\text { Anarcadium occidentale } \\
\text { Stachytarpheta cayennensis }\end{array}$ & 7 & Caju & Pó da folha (espalhado pela ferida) \\
Allium sativum & 6 & Gervão & Pó da folha (espalhado pela ferida) \\
Copaifera spp. & 3 & Alho & Infusão \\
\end{tabular}


onde as populações das áreas rurais desempenham o mesmo tipo de atividade encontrada em Buriticupu/Maranhão.

Percebeu-se que os entrevistados já tinham recebido alguma informação sobre a doença. A grande maioria $(60,6 \%)$ a conhecia como Lésh. Tal desígnio refere-se apenas à região de Buriticupu, podendo ter origem quando do surto epidêmico da doença, ocorrido na área no início de sua colonização (Silva et al.,1979, 1981). Em relação à fonte das informações (informal) obtidas pelos entrevistados, sugere-nos que o programa de controle da LTA, do Ministério da Saúde (MS/FUNASA, 2000), que tem como um dos objetivos específicos "manter a população informada e orientada sobre a doença, reservando espaço para sua participação ativa no desenvolvimento das ações de controle", não está sendo satisfatório nessas áreas.

Apesar de conhecer a doença, a maioria dos entrevistados não soube a forma e local de transmissão, seus métodos profiláticos e curativos. Netto et al. (1985) indicaram em seus resultados que a população de Três Braços/Bahia, detinha um entendimento razoável sobre alguns aspectos epidemiológicos e tratamento da doença, mas demonstraram pouco conhecimento sobre a etiologia e prevenção. Gama et al. (1998) verificaram que em área endêmica de LVA no Estado do Maranhão, os níveis de conhecimento das populações estudadas eram baixos, principalmente em relação à prevenção e terapêutica.

A maioria dos entrevistados não sabia quanto tempo a lesão permanecia ulcerada e o medicamento Glucantime foi referido como o de maior eficácia no tratamento da doença, mesmo com sua conhecida toxicidade. Gama et al. (1998) também concluíram que populações residentes em áreas endêmicas de LVA no Maranhão, apresentavam um baixo nível de conhecimento sobre a terapêutica; entre seus entrevistados apenas sete sabiam do Glucantime como droga para o tratamento da doença. Outros procedimentos usados na ferida foram: sapo torrado, pingo de vela e pólvora, demonstrando que tais métodos são comuns nas áreas endêmicas de LTA no Brasil (Costa, 1986; Folla- dor, 1999; Llanos-Cuentas, 1984). Na Colômbia, Vasquez et al. (1991) relataram que populações rurais aplicam na lesão da leishmaniose produtos como gasolina, petróleo, metal, ácido bórico, ácido acético, pólvora entre outros na tentativa de cicatrizar a lesão.

Como tratamento oficial, alguns medicamentos à base de sulfato de cobre e ácido bórico, já foram utilizados (Werner \& Barreto, 1981). Nas lesões cutâneas produzidas por LTA, os Incas utilizavam o sulfato de arsênio (Dietschy, 1938). O uso de medicamentos caseiros para LTA (lesão cutânea), tem sido utilizado em áreas endêmicas do Peru, Guatemala, Equador e Brasil (Butron-Riveros, 1984; Netto et al., 1985; Torres, 1987).

Algumas plantas foram também citadas no tratamento, às vezes até de forma combinada, sendo seu uso justificado para o alívio da dor. Estudos realizados com plantas já demonstraram alguns resultados contra a doença, o chá de Kalanchoe pinnata quando testado in vivo demonstrou que ratos portadores da lesão por L. amazonensis apresentaram redução do tamanho da mesma, seguida do decréscimo no número de parasitos. Silva et al., (1995). Entre os entrevistados, o limão (Citrus limon) e o gervão (Stachytarpheta cayennensis) foram as plantas mais citadas no tratamento da doença, a forma triturada da folha foi a mais usada. Segundo França et al., (1996) o caju (A. occidentalle), foi utilizado na forma de pó espalhado sobre a lesão, apresentando poder cicatrizante, tendo sido testado em outras doenças, como: hanseníase, sardas, eczemas e verrugas com bons resultados.

Apesar da população ter um conhecimento razoável sobre alguns dos aspectos epidemiológicos da doença, são poucos aqueles que fazem uso de medidas preventivas, contribuindo dessa forma para a manutenção da doença na região.

Pelo exposto, conclui-se que há necessidade da implantação de grupos de notificação da LTA nas localidades ou próximas a elas, e de equipes de educação em saúde para orientar na prevenção e esclarecimentos sobre as formas de transmissão da doença. 


\section{Referências}

AMPUERO, J. S., 1996. Fatores de Risco para a Transmissão de Leishmaniose Cutânea em Crianças de o a Cinco Anos em uma Área Endêmica de Leishmania Viannia braziliensis. Dissertação de Mestrado, Brasília: Núcleo de Medicina Tropical e Nutrição, Universidade de Brasília.

BERMAN, J. D., 1988. Chemotherapy for leishmaniasis: Biochemical mechanisms, clinical efficacy, and future strategies. Reviews of Infectious Diseases, 10:560-586.

BUTRON-RIVEROS, B. C., 1984. Leishmaniasis cutánea andina en el distrito de Surco. Tesis doctoral, Lima: Departamento de Epidemiología, Universidad Peruana Cayetano Heredia.

COSTA, J. M. L., 1986. Estudo Clínico-epidemiológico de um Surto Epidêmico de Leishmaniose Tegumentar Americana em Corte de Pedra-Bahia. Dissertação de Mestrado, Brasília: Núcleo de Medicina Tropical e Nutrição, Universidade Nacional de Brasília.

COSTA, J. M. L.; BALBY, I. A. T.; ROCHA, E. J. S.; SILVA, A. R. S.; REBÊLO, J. M. M.; FERREIRA, L. A.; GAMA, M. E. A.; BRANCO, M. R. F. C.; BURATTINI, M. N. \& SOARES, N. J. S., 1998. Estudo comparativo da leishmaniose tegumentar americana em crianças procedentes das áreas endêmicas de Buriticupu (Maranhão) e Corte de Pedra (Bahia) Brasil. Revista da Sociedade Brasileira de Medicina Tropical, 31:279-288.

COSTA, J. M. L.; TADA, M. S.; NETTO, E. M.; VALE, K. C.; LAGO, E. L. \& MARSDEN, P. D., 1988. Procedência de pacientes portadores de leishmaniose tegumentar americana nas áreas endêmicas de Três Braços e Corte de Pedra - Estado da BahiaBrasil. Revista da Sociedade Brasileira de Medicina Tropical, 21:145-149.

COSTA, J. M. L.; VALE, K. C.; FRANÇA, F; SALDANHA, A. C. R.; SILVA, J. O.; LAGO, E. L.; MARSDEN, P. D.; MAGALHÃES, A. V.; SILVA, C. M. P.; NETO, A. S. \& GALVÃO, C. E. S., 1990. Cura espontânea da leishmaniose causada por Leishmania viannia braziliensis em lesões cutâneas. Revista da Sociedade Brasileira de Medicina Tropical, 23:205-208.

DEAN, J., 1994. Epi Info - Computer Programs for Epidemiology. Atlanta: Division of Surveillance and Epidemiology Studies, Epidemiology Programs Office, Centers for Disease Control and Prevention.

DIETSCHY, H., 1938. Der Arzt in der Inkazeit und seine Mittel. Ciba Zeitschrift, 58:2003-2012.

FOLLADOR, I.; ARAÚJO, C.; CARDOSO, M. A.; TAVARES NETO, J.; BARRAL, A.; NIRANDA, J. C.; BITTENCOURT, A. \& CARVALHO, E. M., 1999. Surto de leishmaniose tegumentar americana em Canoa, Santo Amaro - Bahia - Brasil. Revista da Sociedade Brasileira de Medicina Tropical, 32:497593.

FRANÇA, F.; LAGO, E. L. \& MARSDEN, P. D., 1996. Plants used in the treatment of leishmanial ulcers due to Leishmania (Viannia) braziliensis in the endemic area of Bahia, Brazil. Revista da Sociedade Brasileira de Medicina Tropical, 29:229-232.

GAMA, M. E. A.; BARBOSA, J. S.; PIRES, B.; CUNHA, A. K. B.; FREITAS, A. R.; RIBEIRO, I. R. \& COSTA, J.
M. L., 1998. Avaliação do nível de conhecimento que populações residentes em áreas endêmicas têm sobre leishmaniose visceral, Estado do Maranhão, Brasil. Cadernos de Saúde Pública, 14:381390.

GRIMALDI Jr., G.; TESH, R. B. \& MACMOHON-PRATT, D., 1989. A Review of the geographic distribution and epidemiology of leishmaniasis in the new world. American Journal of Tropical Medicine and Higiene, 41:687-725.

IBGE (Fundação Instituto Brasileiro de Geografias e Estatística), 1984. Atlas do Maranhão. Rio de Janeiro: Governo do Maranhão/IBGE.

LLANOS-CUENTAS, E. A., 1984. Estudo Clínico Evolutivo da Leishmniose em Área Endêmica de Leishmnia Braziliensis Braziliensis Três Braços-Bahia, Brasília. Dissertação de Mestrado, Brasília: Núcleo de Medicina Tropical e Nutrição, Universidade de Brasília.

MARSDEN, P., 1986. Mucosal leishmaniasis ("Espundia" Escomel, 1911). Transactions of the Royal Society of Tropical Medicine and Hygiene, 80:859875.

MOREIRA, R. C. R.; COSTA, J. M. L.; SALDANHA, A. C. \& SILVA, A. R., 1998. Projeto Buriticupu Maranhão II. Plantas usadas como terapêutica da leishmaniose tegumentar americana na região de Buriticupu - Maranhão. Revista da Sociedade Brasileira de Medicina Tropical, 31(Sup. 1):126.

MS (Ministério da Saúde)/FUNASA (Fundação Nacional de Saúde), 2000. Manual de Controle da Leishmaniose Tegumentar Americana. Brasília: Centro Nacional de Epidemiologia, FUNASA, MS.

NETTO, E. M.; TADA, M. S.; GOLIGHTLY, L.; KALTER, D. C.; LAGO, E. L.; BARRETO, A. I. R. C. \& MARSDEN, P. D., 1985. Conceitos de uma população local a respeito da leishmaniose mucocutânea em uma área endêmica. Revista da Sociedade Brasileira de MedicinaTropical, 18:33:37.

PESSOA, S. B. \& BARRETO, M. P., 1948. Leishmaniose Tegumentar Americana. Rio de Janeiro: Imprensa Nacional/São Paulo: Serviço de Parasitologia, Departamento de Medicina, Faculdade de São Paulo.

REBÊLO, J. M. M.; OLIVEIRA, S. T.; BARROS, V. L. L.; SILVA, F. S.; COSTA, J. M. L.; FERREIRA, L. A. A. \& SILVA, A. R., 2000. Plebotominae (Diptera: Psychodidae) de Lagoas, município de Buriticupu, Amazônia Maranhense. I - Riqueza e abundância relativa das espécies em área de colonização recente. Revista da Sociedade Brasileira de Medicina Tropical, 33:11-19.

ROCHA, F. A.; OLIVEIRA, M. F.; NOGUEIRA, L. \& FREITAS, C. E. J., 1991. Estudo epidemiológico dos casos de leishmaniose visceral (calazar) registrados no Hospital São José, em 1980. Fortaleza - Ceará. Revista Brasileira de Clínicas, 23:87-92.

SILVA, A. R.; MARTINS, G.; MELO, J. E. M.; ARAÚJO, J. P. \& MENDES, M. G., 1979. Surto epidêmico de leishmaniose tegumentar americana ocorrido na colonização agrícola de Buriticupu (Estado do Maranhão), Brasil. Revista do Instituto de Medicina Tropical de São Paulo, 21:43-50.

SILVA, A. R.; MENDES, J. R.; RODRIGUES, M. J.; CARVALHO, Z. S.; REIS, F. M. P.; MELO, J. E. M. \& 
MORAES, J. C. O., 1981. Leishmaniose cutânea difusa (LCD). Registro de um caso em Buriticupu, Estado do Maranhão, Brasil. Revista do Instituto de Medicina Tropical de São Paulo, 23:31-35.

SILVA, S. A. G.; COSTA, S. S.; MENDONÇA, S. C. F.; SILVA, E. M.; MORAES, V. L. G.\& ROSSI-BERGMANN, B., 1995. Therapeutic and effect of oral Kalanchoe pinnata leaf extract in murine leismaniasis. Acta Tropica, 60:201-210.

TORRES, M. F., 1987. Current situation in regard to leishmaniasis in Guatemala. In: International Workshop on Control Strategies for the Leismaniases, Proceedings, Otawa: World Health Organization.
VAZQUEZ, M. L.; KROEGER, A.; LIPOWSKY, R. \& ALZATE, A., 1991. Conceitos populares sobre la Leishmaniasis cutánea en Colombia y su aplicabilidad en programas de control. Boletín Epidemiológico de la Organización Panamericana de la Salud, 110:402-415.

WERNER, J. K. \& BARRETO, P., 1981. Leishmaniasis in Colombia: A review. American Journal of Tropical Medicine and Higiene, 41:687-725.

WHO (World Health Organization), 1990. Control of the Leishmaniasis. Techinical Report Series 793. Geneva:WHO.

Recebido em 3 de agosto de 2000

Versão final reapresentada em 17 de abril de 2001

Aprovado em 7 de novembro de 2001 